\title{
Theoretical and Experimental Essentials on Baryon Form Factors
}

\author{
Monica Bertani ${ }^{1} @$, Alessio Mangoni ${ }^{2} @$ and Simone Pacetti ${ }^{2,3, *}$ \\ 1 INFN Laboratori Nazionali di Frascati, 00044 Frascati, Italy; monica.bertani@Inf.infn.it \\ 2 INFN Sezione di Perugia, 06100 Perugia, Italy; alessio.mangoni@pg.infn.it \\ 3 Dipartimento di Fisica e Geologia, Università di Perugia, 06100 Perugia, Italy \\ * Correspondence: simone.pacetti@unipg.it
}

check for updates

Citation: Bertani, M.; Mangoni, A.; Pacetti, S. Theoretical and

Experimental Essentials on Baryon Form Factors. Symmetry 2022, 14, 439 https://doi.org/10.3390/ sym14030439

Academic Editor: Sergei D. Odintsov

Received: 4 January 2022

Accepted: 11 February 2022

Published: 23 February 2022

Publisher's Note: MDPI stays neutral with regard to jurisdictional claims in published maps and institutional affiliations.

Copyright: () 2022 by the authors Licensee MDPI, Basel, Switzerland. This article is an open access article distributed under the terms and conditions of the Creative Commons Attribution (CC BY) license (https:// creativecommons.org/licenses/by/ $4.0 /)$.

\begin{abstract}
This brief review is a practical guide on the basic concepts, pivotal formulae, standard relations, and some unusual, often forgotten, but sometimes revealing features of baryons' form factors. All available measured values of nucleon form factors in the space-like and time-like regions extracted from scattering and annihilation cross-sections are also reported.
\end{abstract}

Keywords: form factor; baryons; polarization; analyticity

\section{Introduction}

The study of baryons is one of the greatest challenges in particle physics (see, e.g., [1]). It is fundamental for a deep understanding of the dynamical mechanisms that rule quantum chromodynamics, the $Q C D$, at energy regimes where the perturbative character of this gauge theory is still not effective because of the so-called phenomenon of asymptotic freedom.

Baryons, unlike elementary particles, have an internal structure of three valence quarks surrounded by a dynamical and intensely interacting sea of virtual quark-antiquark pairs and gluons.

From the theoretical point of view and in the framework of quantum field theory, the most general description of the mechanisms that underlie and, hence, rule baryon dynamics is grounded on the concept of form factors (FFs). They represent energy-dependent coupling constants that parametrize the baryon four-currents and encode all the information concerning their dynamics.

Formally, the electromagnetic (EM) FFs have to be introduced to obtain the most general, gauge-, and Lorentz-invariant amplitude of the non-point-like baryon-photon vertex $B B \gamma$, being, indeed, Lorentz scalar functions of $q^{2}$, the squared four-momentum of the virtual photon.

Experimental values of the FFs in the space-like and time-like regions are extracted from the data on the cross-sections of two main processes: the electron-baryon scattering and the annihilations: either electron-positron into baryon-anti-baryon or the time-reversed process baryon-anti-baryon into lepton-anti-lepton. Since the FFs parametrize the singlephoton vertex $B B \gamma$, the amplitudes of these processes have to be considered in the Born approximation, i.e., at first order, so that the corresponding Feynman diagrams are those shown in Figures 1 and 2 for the scattering and the annihilations, respectively.

In principle, the two Lorentz scalar degrees of freedom, in terms of which the vertex amplitude has to be defined at each $q^{2}$, can be expressed by an arbitrary pair of independent FFs. Nevertheless, some pairs can be preferred with respect to others on the basis of specific convenience criteria. One of these is represented by the pair of the so-called electric, $G_{E}\left(q^{2}\right)$, and magnetic, $G_{M}\left(q^{2}\right)$, Sachs FFs. Their peculiarity consists of having Fourier transforms that, in the reference system where there is no energy exchange (Breit frame), coincide 
with the spatial densities of the electric charge and the magnetic moment of the baryon. The Sachs FFs are the combinations:

$$
\begin{aligned}
& G_{E}\left(q^{2}\right)=F_{1}\left(q^{2}\right)+\tau F_{2}\left(q^{2}\right), \quad \tau=\frac{q^{2}}{4 m^{2}} \\
& G_{M}\left(q^{2}\right)=F_{1}\left(q^{2}\right)+F_{2}\left(q^{2}\right),
\end{aligned}
$$

where $F_{1}\left(q^{2}\right)$ and $F_{2}\left(q^{2}\right)$ are the Dirac and Pauli FFs and $m$ is the mass of the baryon.

\subsection{The Space-Like Region}

In the space-like region, namely for $q^{2}<0$, the FF values are extracted from the cross-section data on the scattering processes:

$$
e^{ \pm} B \rightarrow e^{ \pm} B
$$

whose Feynman diagram, at the lowest order, i.e., in the Born approximation, is shown in Figure 1.

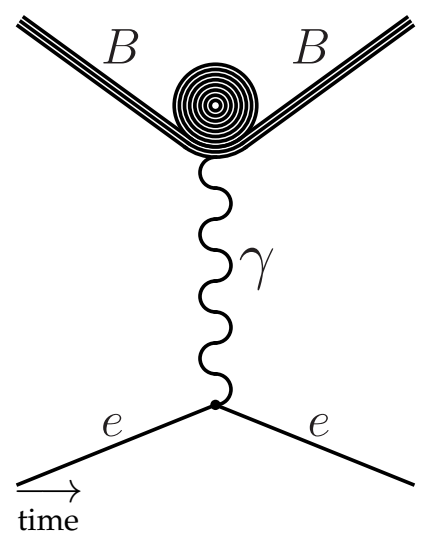

Figure 1. Feynman diagram in the one-photon exchange approximation for the electron-baryon scattering $e B \rightarrow e B$.

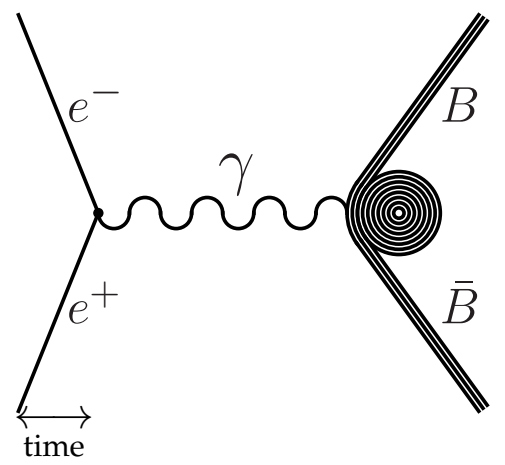

Figure 2. Feynman diagram, at first order, for the $e^{+} e^{-} \leftrightarrow B \bar{B}$ annihilation.

Of course, given the fact that neither stable targets, nor beams of strange, charm, and bottom baryons are available, the scattering process is exploitable as a tool to investigate the FFs only for the nucleons, i.e., for $B=p$ and $n$. 


\subsection{The Time-Like Region}

In the time-like region, and solely above the physical threshold, i.e., for $q^{2}>(2 m)^{2}$, $m$ being the baryon mass, the moduli of the FFs can be extracted from the data on the cross-section of the annihilation processes:

$$
e^{+} e^{-} \leftrightarrow B \bar{B} .
$$

The Feynman diagram representing the one-photon exchange reaction is shown in Figure 2. It represents just the crossed reaction of that of the diagram in Figure 1.

\subsection{The Nucleons}

Concerning the nucleons, which for obvious reasons represent the most studied baryons, the first pioneering measurement was that of the space-like proton FF. It dates back to 1955 when Hofstadter and McAllister [2] published their work on electron-proton scattering. Only three years later, in 1958, also the neutron FF was measured [3], and even in this case, the experimental investigation concerned its space-like values. The innovative experiment conceived of and realized by Yearian and Hofstadter [3] consisted of measuring the cross-section of the scattering of an electron beam impinging on a deuteron target. This also allowed extracting for the first time the root-mean-squared radius of the deuteron.

Since then and especially in the last two decades, a growing number of experiments have been providing more and more sets of data that, with high accuracy and covering all kinematic regions, are going to complete, piece by piece, the complex puzzle of baryon FFs.

\section{Theoretical Aspects}

\subsection{Scattering}

Concerning spin- $1 / 2$ baryons, the Feynman amplitude in the Born approximation for the scattering process:

$$
e^{-}\left(k_{1}\right) B\left(p_{1}\right) \rightarrow e^{-}\left(k_{2}\right) B\left(p_{2}\right)
$$

with reference to the Figure 2, where in parentheses are shown the particles four-momenta, can be written as:

$$
\mathcal{A}_{e B \rightarrow e B}=-\frac{i}{q^{2}} l^{\mu} J_{\mu}
$$

$q=p_{2}-p_{1}=k_{1}-k_{2}$ being the four-momentum of the photon and where $l^{\mu}$ and $J^{\mu}$ are the leptonic and baryonic currents. The former is related to a point-like vertex and, hence, has the typical form:

$$
l^{\mu}=e \bar{u}\left(k_{1}\right) \gamma^{\mu} u\left(k_{2}\right) .
$$

The baryon current, instead, refers to a structured vertex, the baryon itself being a non-elementary particle. It follows that it has to be written in the most general Lorentz and gauge-invariant form, i.e., accounting for two scalar degrees of freedom, which depend only on $q^{2}$ and cannot be predicted by the theory. They are represented by a pair of FFs, which are Lorentz scalar functions of $q^{2}$. As already stated in the previous section, even though there are infinite pairs of independent FFs that can be used, some of them are indeed preferable. Besides the pair of the Sachs FFs, one of the most suitable choices is represented by the Dirac and Pauli FFs, $F_{1}\left(q^{2}\right)$ and $F_{2}\left(q^{2}\right)$. In terms of these FFs, the baryonic current reads:

$$
J^{\mu}=e \bar{u}\left(p_{1}\right) \Gamma^{\mu} u\left(p_{2}\right)=e \bar{u}\left(p_{1}\right)\left[F_{1}\left(q^{2}\right) \gamma^{\mu}-\frac{\sigma^{\mu v} q_{v}}{2 m} F_{2}\left(q^{2}\right)\right] u\left(p_{2}\right),
$$


where:

$$
\sigma^{\mu \nu}=\frac{\gamma^{\mu} \gamma^{\nu}-\gamma^{v} \gamma^{\mu}}{2}=\frac{\left[\gamma^{\mu}, \gamma^{v}\right]}{2}
$$

The tensor component of $J^{\mu}$ is strictly related to the non-elementary nature of the baryon. In fact, the expression of the point-like current, namely the one of Equation (3), is obtained by considering a constant and unitary Dirac FF and a null Pauli FF, i.e., with: $F_{1}\left(q^{2}\right)=1$ and $F_{2}\left(q^{2}\right)=0$.

\subsection{Annihilation}

In the case of annihilation, the two possible processes are:

$$
e^{+}\left(k_{1}\right) e^{-}\left(k_{2}\right) \leftrightarrow \gamma^{*}(q) \leftrightarrow B\left(p_{1}\right) \bar{B}\left(p_{2}\right) .
$$

The corresponding unique Feynman diagram in the Born approximation is shown in Figure 2. Under the aegis of crossing symmetry, the amplitude of the one-photon-exchange process, in the case of $B \bar{B}$ production, has the same form of that of Equation (2) regarding the scattering process, i.e.,

$$
\mathcal{A}_{e^{+} e^{-} \rightarrow B \bar{B}}=-\frac{i}{q^{2}} l^{\mu} J_{\mu}
$$

In this kinematic region, the photon four-momentum $q^{\mu}=k_{1}^{\mu}+k_{2}^{\mu}=p_{1}^{\mu}+p_{2}^{\mu}$ is a time-like four-vector, in fact $q^{2} \geq(2 m)^{2}>0, m$ being the baryon mass, while the leptonic and baryonic four-currents are:

$$
l_{\mu}=e \bar{u}\left(k_{1}\right) \gamma^{\mu} v\left(k_{2}\right), \quad J^{\mu}=e \bar{u}\left(p_{1}\right) \Gamma^{\mu} v\left(p_{2}\right),
$$

with $\Gamma^{\mu}$ given in Equation (4).

By using the amplitude of Equation (5) and the baryonic current parametrized in terms of the Sachs FFs of Equation (1), the expression of the differential cross-section for the unpolarized annihilation process $e^{+} e^{-} \rightarrow B \bar{B}$ in the center of mass frame reads:

$$
\frac{d \sigma}{d \Omega}=\frac{\alpha^{2} \beta C\left(q^{2}\right)}{4 q^{2}}\left(\left|G_{E}\left(q^{2}\right)\right|^{2} \frac{\sin ^{2}(\theta)}{\tau}+\left|G_{M}\left(q^{2}\right)\right|^{2}\left(1+\cos ^{2}(\theta)\right)\right),
$$

where $\beta=\sqrt{1-1 / \tau}$ is the velocity of the baryon, $\theta$ is the scattering angle, $\alpha=e^{2} /(4 \pi)$ is the fine-structure constant, and:

$$
C\left(q^{2}\right)=\left\{\begin{array}{ll}
1 & \text { for neutral baryons } \\
\frac{\pi \alpha / \beta}{1-e^{-\pi \alpha / \beta}} & \text { for charged baryons }
\end{array},\right.
$$

is the Sommerfeld-Gamow correction factor accounting for the electromagnetic interaction between the final-state baryon and anti-baryon.

The differential cross-section, which is obviously defined for $q^{2}>(2 m)^{2}$, i.e., above the so-called physical threshold, depends on the moduli of FFs. Usually, owing to the impossibility of making statistically significant angular distribution studies, only an effective FF, instead of the single moduli of the two Sachs FFs, is extracted from the cross-section data. The effective FF is defined as:

$$
G_{\text {eff }}\left(q^{2}\right)=\sqrt{\frac{\left|G_{E}\left(q^{2}\right)\right|^{2}+2 \tau\left|G_{M}\left(q^{2}\right)\right|^{2}}{1+2 \tau}},
$$


in such a way that the total cross-section, obtained by integrating the expression of Equation (6), at a given $q^{2}$, is proportional to $G_{\text {eff }}^{2}\left(q^{2}\right)$, being:

$$
\sigma\left(q^{2}\right)=\frac{4 \pi \alpha^{2} \beta C\left(q^{2}\right)}{3 q^{2}}\left(\frac{1}{2 \tau}+1\right) G_{\text {eff }}^{2}\left(q^{2}\right) .
$$

\subsection{Properties}

From the theoretical point of view, FFs are analytic complex functions of $q^{2}$ with the branch cut $\left(q_{\mathrm{th}}^{2}, \infty\right)$ along the positive real axis, which opens at the so-called theoretical threshold $q_{\mathrm{th}}^{2}$ and goes up to infinity. It represents the mass squared of the lightest hadronic intermediate channel that can couple directly with the virtual photon and the baryonic final state. In general, the pions being the lightest hadrons, this intermediate state is either a two- or a three-pion system, depending on the isospin of the final state. In fact, the virtual photon, owning to both the isoscalar and the isovector components, does not select this quantum number. In particular, in the case of a pure isovector final state, only intermediate states with an even number of pions are allowed, hence $q_{\mathrm{th}}^{2}=\left(2 m_{\pi}\right)^{2}$, while in the case of a pure isoscalar, $q_{\mathrm{th}}^{2}=\left(2 m_{\pi}+m_{\pi^{0}}\right)^{2}$, where $M_{\pi}$ and $m_{\pi^{0}}$ are the masses of the charged and neutral pions, respectively. Of course, if the final state had both the components, the branch point would be that of the two-pion, i.e., of the isovector component, because it lies at a lower $q^{2}$.

Exploiting scattering and annihilation processes involving leptons and the same baryons, it is possible to measure the FFs in both the space-like region, where they are real functions, and the time-like region, where, starting from the branch point $q_{\mathrm{th}^{\prime}}^{2}$ they have a non-null imaginary part as a consequence of the optical theorem.

More in detail, FFs are experimentally accessible only in the portion of the time-like region, starting at the physical threshold of the $B \bar{B}$ system, i.e., $q_{\text {phys }}^{2}=(2 m)^{2}$, where $m$ is the baryon mass, well above the theoretical threshold. Moreover, in this limited $q^{2}$ interval, only the moduli of the FFs are accessible. Information about their relative phase are indeed attainable solely by taking advantage of polarization observables.

It is quite interesting to notice that, even though FFs are measurable only in the portion of the time-like region with $q^{2}>q_{\text {phys }}^{2}$, analyticity implies that they are well defined at each $q^{2}$ not belonging to the branch cut and, hence, also in the interval $\left(0, q_{\text {phys }}^{2}\right)$, namely at those $q^{2}$ values lying on the upper edge of the branch cut with $\operatorname{Re}\left(q^{2}\right) \in\left(0, q_{\text {phys }}\right)$. It is just for this reason, i.e., the fact of hosting well-defined FFs, which, nevertheless, are not experimentally accessible, that it is called the unphysical region.

Analytic continuation techniques can be used not only to connect FF values related to different $q^{2} \mathrm{~s}^{\prime}$, but also and mainly to extend their definition in $q^{2}$-regions, which are still experimentally unexplored or even inaccessible.

One of these analytic continuation techniques, which is particularly suitable to handle the FFs, is that based on the dispersion relations. The most used dispersion relation is represented by the following integral expression:

$$
G\left(q^{2}\right)=\frac{1}{\pi} \int_{q_{\mathrm{th}}^{2}}^{\infty} \frac{\operatorname{Im}[G(s+i \epsilon)]}{s-q^{2}} d s .
$$

It allows computing the value of the analytic function $G\left(q^{2}\right)$ at a generic $q^{2}$ belonging to its analyticity domain, e.g., as in the case of an FF, the $q^{2}$ complex plane with the branch cut $\left(q_{\mathrm{th}}^{2}, \infty\right)$, by knowing solely the imaginary part on the upper edge of the branch cut.

As an example, among a variety of possible applications, a model for the analytic continuation of nucleon electromagnetic FFs from the space-like to the time-like region was the subject of [4]. 
Finally, the asymptotic behavior of FFs can be inferred by making simple considerations in the framework of perturbative QCD (pQCD). In particular, in the space-like region, we obtain the power-law asymptotic behavior:

$$
G_{E, M}\left(q^{2}\right) \sim\left(-q^{2}\right)^{-2}, \quad q^{2} \rightarrow-\infty
$$

The narrowness of FFs at finite values of $q^{2}$, as well as their regularity at infinity, allow applying the Phragmén-Lindelöf theorem [5], which extends the space-like asymptotic behavior, not only to the time-like region, that is for $q^{2}$, which diverges along the positive real axis, but also to a divergence in any direction in the complex plane. It follows that, by considering the polar representation $q^{2}=\left|q^{2}\right| e^{i \arg \left(q^{2}\right)}$, we have:

$$
G_{E, M}\left(q^{2}\right) \sim\left(q^{2}\right)^{-2}, \quad\left|q^{2}\right| \rightarrow \infty
$$

uniformly in $\arg \left(q^{2}\right)$, i.e., $\forall \arg \left(q^{2}\right) \in(0,2 \pi)$, where $\arg \left(q^{2}\right) \rightarrow 0^{+}$and $\arg \left(q^{2}\right)=\pi$ represent the time-like and the space-like regions, respectively.

\subsection{Radii}

As already discussed, in the so-called Breit frame, Sachs FFs $G_{E}$ and $G_{M}$ represent the Fourier transforms of the spatial distributions of electric charge and magnetic momentum, respectively. In light of that, they contain information on the corresponding sizes of the baryon, namely on the spatial extension of the volume in which a certain percentage of the total electric charge and magnetic moment is distributed. Such a piece of information is coded in the root-mean-squared radius, which is defined by the zero-four-momentum transferred limit either of the normalized first derivative of the FF or, equivalently, of the logarithmic one, i.e.,

$$
\left\langle r_{E, M}^{2}\right\rangle=\lim _{q^{2} \rightarrow 0} 6 \frac{d \ln G_{E, M}}{d q^{2}}=\frac{6}{G_{E, M}(0)} G_{E, M}^{\prime}(0) .
$$

As widely discussed (see, e.g., [6] and the references therein), the extraction of this value from the data represents a quite serious challenge, because it is founded on two steps performed at the borderlines of the validity domains of the assumptions in which the FFs definitions are based. Firstly, data on the FFs have to be extrapolated at $q^{2}=0$, in the limit where the scattering becomes a capture process; secondly, the variation, i.e., the first derivative of the data has to be computed.

A further constraint on the extraction of the root-mean-squared radius from the FFs data is provided by the dispersion relations, in particular from the those of the modulus. In fact, in terms of such a powerful mathematical tool, it is possible to relate a purely static property of the baryons to an extensive and, hence, more reliable observable, at least from the experimental point of view, namely their moduli over the time-like branch cut. The dispersion relations for the modulus [7] are defined in terms of the logarithm of the FFs as:

$$
\ln \left(G_{E, M}\left(q^{2}\right)\right)=\frac{\sqrt{q_{\mathrm{th}}^{2}-q^{2}}}{\pi} \int_{q_{\mathrm{th}}^{2}}^{\infty} \frac{\ln \left|G_{E, M}(s+i \epsilon)\right|}{\left(s-q^{2}\right) \sqrt{s-q_{\mathrm{th}}^{2}}} d s,
$$

for all $q^{2} \notin\left(q_{\mathrm{th}}^{2}, \infty\right)$. It follows that the root-mean-squared radii are given by:

$$
\left\langle r_{E, M}^{2}\right\rangle=\frac{3}{\pi \sqrt{q_{\mathrm{th}}^{2}}} \int_{q_{\mathrm{th}}^{2}}^{\infty} \frac{\ln \left|G_{E, M}(s+i \epsilon)\right|}{s^{2} \sqrt{s-q_{\mathrm{th}}^{2}}} d s,
$$

and hence, the knowledge of the FFs' moduli in the whole time-like interval, corresponding to the upper edge of the branch cut, running from the theoretical threshold $q_{\text {th }}^{2}$ up to infinity, would allow computing $\left\langle r_{E, M}^{2}\right\rangle$ as an integral instead of as a punctual derivative at $q^{2}=0$. 


\section{Polarization Variables}

The polarization of the baryons and anti-baryons produced in electron-positron annihilation processes, when the initial leptons are unpolarized, is a clear and univocal consequence of the complex nature of things.

In the case of hyperons, the polarization can be easily inferred by taking advantage of their self-analyzing weak decays. Instead, in the case of nucleons, which are stable particles, the polarization can be only measured directly by means of detectors equipped with polarimeters.

More in detail, by considering the annihilation processes $e^{+} e^{-} \rightarrow B \bar{B}$ and defying the $x z$ as the scattering plane, the $y$-component of the polarization vector of the outgoing baryon in the $B \bar{B}$ center of mass frame is:

$$
\mathcal{P}_{y}=-\frac{\sqrt{\tau}\left|G_{E} G_{M}\right| \sin (2 \theta) \sin \left(\arg \left(G_{E} / G_{M}\right)\right)}{\tau\left|G_{M}\right|^{2}\left(1+\cos ^{2}(\theta)\right)+\left|G_{E}\right|^{2} \sin ^{2}(\theta)} .
$$

It is proportional to $\sin \left(\arg \left(G_{E} / G_{M}\right)\right)$, and it is null in the case of real FFs. This is because if $G_{E}$ and $G_{M}$ are real, their relative phase $\arg \left(G_{E} / G_{M}\right)$ is an integer multiple of $\pi$ radians, hence $\sin \left(\arg \left(G_{E} / G_{M}\right)\right)=0$ and, obviously, $\mathcal{P}_{y}=0$.

It is interesting to notice that the knowledge of the polarization vector of a single final baryon allows extracting only the sinus of the relative phase, and hence, its value is known in the form $\arg \left(G_{E} / G_{M}\right) \pm k \pi$, with $\arg \left(G_{E} / G_{M}\right) \in[-\pi / 2, \pi / 2]$ and $k$ being an integer that defines the determination of the relati ve phase. As was proven in [8], such a determination plays a crucial role because it embodies unique information about the asymptotic behavior of FFs, as well as the presence of zeros for the electric FF in the space-like region.

Recently, successful attempts [9] of extracting information on the relative phase $\arg \left(G_{E}^{\Lambda} / G_{M}^{\Lambda}\right)$ and also the modulus of the FFs ratio have been made by the BESIII Collaboration. The process that has been studied is the annihilation $e^{+} e^{-} \rightarrow \Lambda\left(p \pi^{-}\right) \bar{\Lambda}\left(\bar{p} \pi^{+}\right)$, where the final baryons (anti-baryons) are identified through their self-analyzing weak decay in protons (anti-protons) and negative (positive) pions.

\section{Transition FFs}

The study of electric and magnetic transition FFs of the baryons is another important step for a better understanding of their structure.

Having different baryons and hence different masses gives the possibility of measuring the transition FFs in kinematic regions that are not accessible in the case of a usual baryonanti-baryon pair. As an example, we can consider the transition FFs of the neutral $\Lambda$ and $\Sigma^{0}$ hyperons, which can be accessed experimentally for $q^{2} \in\left[\left(2 m_{e}\right)^{2},\left(m_{\Sigma}-m_{\Lambda}\right)^{2}\right], m_{e}$ being the electron mass, by studying the decay $\Sigma^{0} \rightarrow \Lambda e^{+} e^{-}$. Following [10], the matrix element for the transition $\Sigma^{0} \bar{\Lambda} \rightarrow \gamma^{*}$ can be written as:

$$
\left\langle 0\left|J^{\mu}\right| \Sigma^{0} \bar{\Lambda}\right\rangle=e \bar{v}_{\Lambda}\left[\left(\gamma^{\mu}+\frac{m_{\Lambda}-m_{\Sigma}}{q^{2}} q^{\mu}\right) F_{1}\left(q^{2}\right)-\frac{i \sigma^{\mu v} q_{v}}{m_{\Lambda}+m_{\Sigma}} F_{2}\left(q^{2}\right)\right] u_{\Sigma},
$$

where the $F_{1}$ and $F_{2}$ are the Dirac and Pauli transition FFs. They are related to the electric and the magnetic ones, $G_{E}$ and $G_{M}$, through the relations:

$$
\begin{aligned}
G_{E}\left(q^{2}\right) & =F_{1}\left(q^{2}\right)+\frac{q^{2}}{\left(m_{\Lambda}+m_{\Sigma}\right)^{2}} F_{2}\left(q^{2}\right), \\
G_{M}\left(q^{2}\right) & =F_{1}\left(q^{2}\right)+F_{2}\left(q^{2}\right),
\end{aligned}
$$

representing the homologous of those of Equation (1), but for a pair of different baryon and anti-baryon. 
Moreover, from the rate of the radiative decay $\Sigma^{0} \rightarrow \Lambda \gamma$, it is possible to extract the value of $G_{M}(0)[10]$ as:

$$
G_{M}(0)=\sqrt{\frac{8 \pi m_{\Sigma}^{3}\left(m_{\Lambda}+m_{\Sigma}\right)^{2} \Gamma\left(\Sigma^{0} \rightarrow \Lambda \gamma\right)}{e^{2}\left(m_{\Sigma}^{2}-m_{\Lambda}^{2}\right)^{3}}}
$$

\section{Experimental Results}

The experimental values of the proton effective FF (time-like region) [11,12], obtained by various collaborations, are shown in Figure 3. Recently, the BESIII Collaboration studied the process $e^{+} e^{-} \rightarrow p \bar{p}(\gamma)$ [13], using both procedures of the energy scan from $\sqrt{s}=2.00 \mathrm{GeV}$ up to $\sqrt{s}=3.08 \mathrm{GeV}$ and the initial state radiation. For the first time ever, in the case of the proton, the high number of collected events allowed extracting moduli of single FFs $\left|G_{E}\right|$ and $\left|G_{M}\right|$, whose data are shown in Figure 4. Figure 5 shows the modulus of the ratio $G_{E} / G_{M}$.

Apart from that of the moduli of single FFs, which still do not have a precedent, the total cross-section $\sigma\left(e^{+} e^{-} \rightarrow p \bar{p}\right)$ measured by the BESIII experiment is consistent with previous experimental investigations. Concerning the modulus of the ratio $G_{E} / G_{M}$, as shown in Figure 5, the BESIII data, in agreement with the BABAR ones, highlight the well-known discrepancy with the first measurement of such an observable performed by the PS170 in the reversed process $p \bar{p} \rightarrow e^{+} e^{-}$.

For a theoretical overview of proton FFs and hints for phenomenological interpretations of the data, see [14].

Concerning the process $e^{+} e^{-} \rightarrow n \bar{n}$, BESIII measured the cross-section at 18 energy points from $2.00 \mathrm{GeV}$ up to $3.08 \mathrm{GeV}$ [15]. The data are shown in Figure 6 together with other datasets. As discussed in [15], BESIII measurements are systematically below all other previously measured values above $0.1 \mathrm{GeV}$ (see Figure 6), while still in agreement within two standard deviations, taking into account individual uncertainties. FENICE results on the Born cross-section show an unexpected ratio:

$$
R_{n p}=\frac{\sigma\left(e^{+} e^{-} \rightarrow n \bar{n}\right)}{\sigma\left(e^{+} e^{-} \rightarrow p \bar{p}\right)}>1
$$

indicating a stronger coupling of the virtual photon with the neutron than with the proton. BESIII has tested the ratio of the cross-sections (see Figure 7), showing that $R_{n p}$ is instead smaller than one at all energies, as expected by various theoretical predictions. In Figure 7 , we show, as a horizontal reference line, the squared ratio between the neutron and proton magnetic momenta, since for large $q^{2}$, the cross-section is dominated by the square modulus of the magnetic form factor, which is normalized to the magnetic momentum at $q^{2}=0$.

With reference to other baryons in the final state, Figure 8 shows the cross-section $e^{+} e^{-} \rightarrow B \bar{B}$, where $B \bar{B}=\Lambda_{c} \bar{\Lambda}_{c}, \Lambda \bar{\Sigma}^{0}+$ c.c. $, \Sigma^{0} \bar{\Sigma}^{0}, \Lambda \bar{\Lambda}, \Xi^{0} \bar{\Xi}^{0}, \Xi^{-} \bar{\Xi}^{+}, \Sigma^{-} \bar{\Sigma}^{+}, \Sigma^{+} \bar{\Sigma}^{-}$, measured by the BESIII, BABAR, and DM2 experiments. Some of this channel is treated in the other papers of this Special Issue "Baryon Structure: Form Factors and Polarization". 


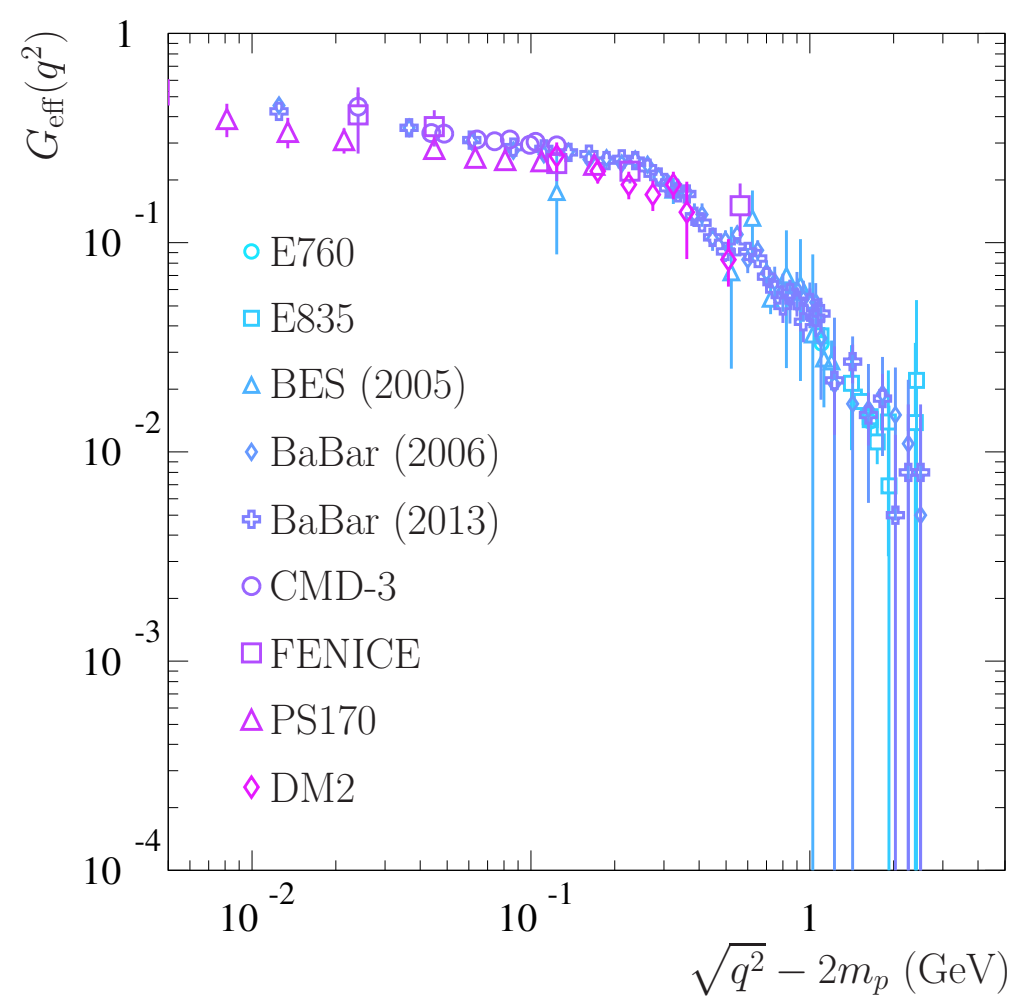

Figure 3. Moduli of the proton effective FF, $G_{\text {eff }}\left(q^{2}\right)$, measured by the experiments: E760 [16], E835 [17,18], BES [19], BABAR [20,21], CMD-3 [22], FENICE [23], PS170 [24], and DM2 [25,26].

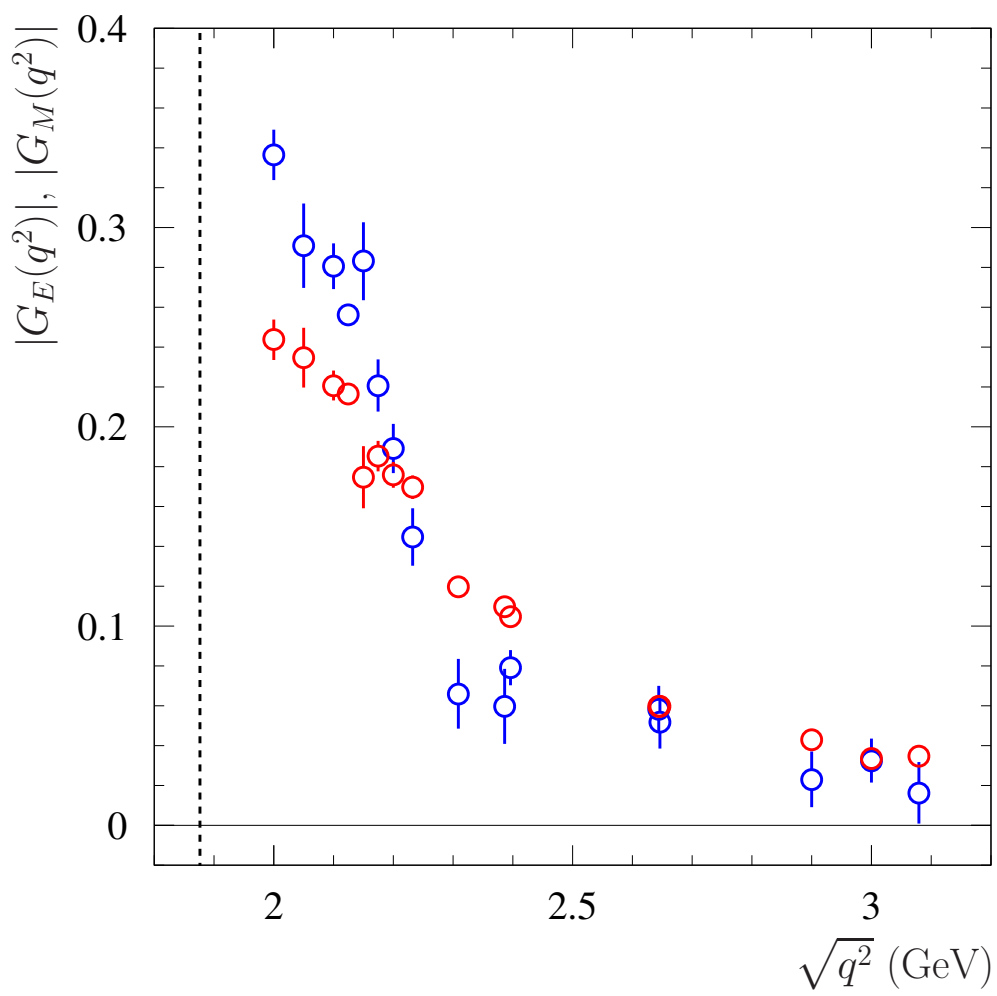

Figure 4. Experimental values for the modulus of the electric (blue circles) and magnetic (red circles) proton FFs, $G_{E}$ and $G_{M}$, measured by the BESIII Collaboration in 2019 [13]. The dashed line indicates the production threshold, $\sqrt{q^{2}}=2 m_{p}$, where $m_{p}$ is the proton mass. 


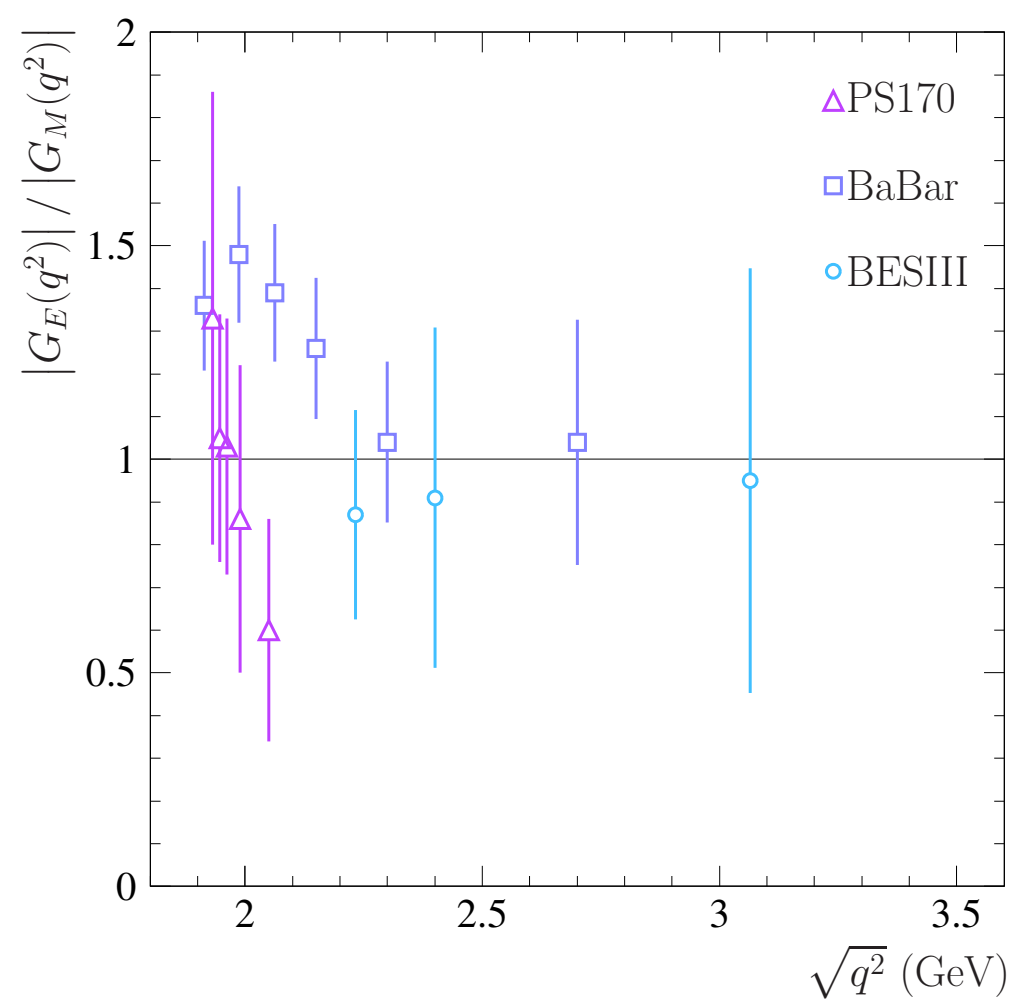

Figure 5. Experimental values for the moduli of the proton FFs' ratio, $\left|G_{E} / G_{M}\right|$, measured by the experiments: BESIII Collaboration in 2019 [13], BABAR [21], and PS170 [24].

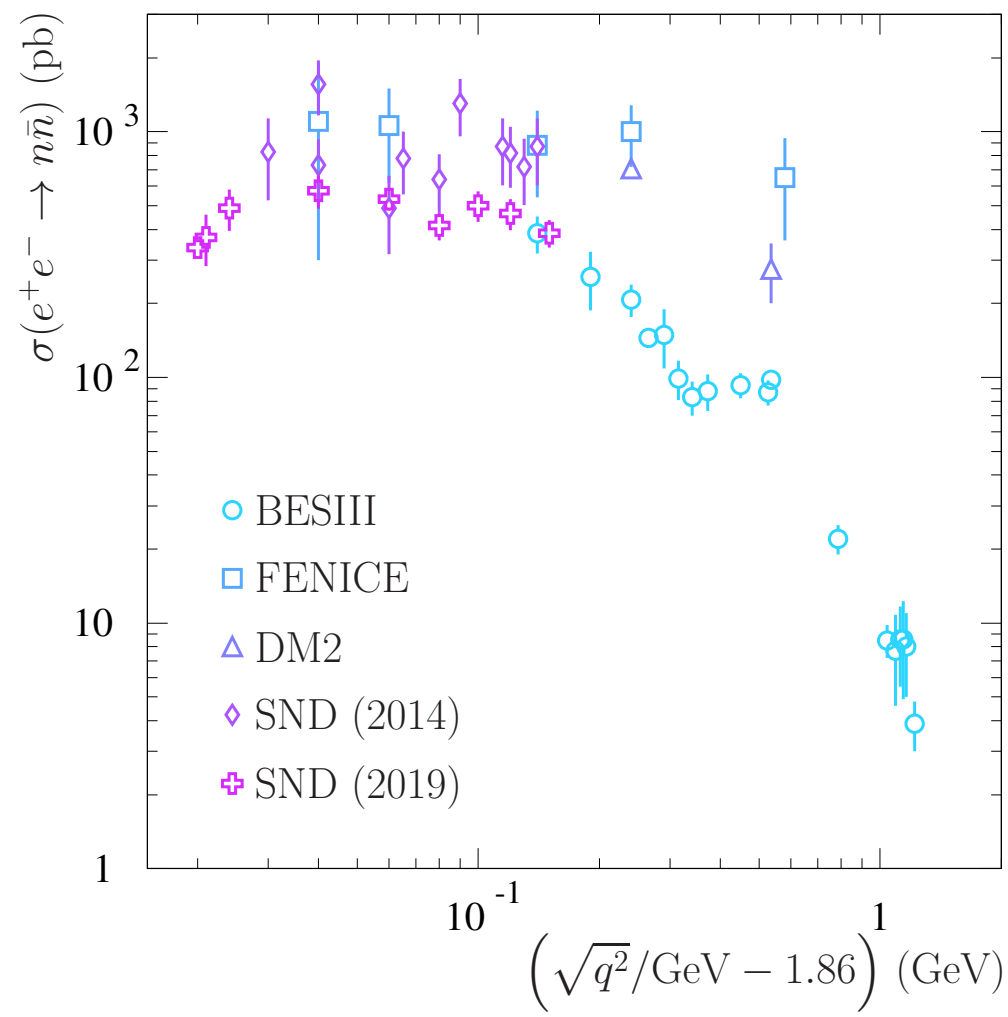

Figure 6. Data on the $e^{+} e^{-} \rightarrow n \bar{n}$ cross-section measured by the experiments: BESIII [15], FENICE [27], DM2 [28], SND (2014) [29], and SND (2019) [30]. 


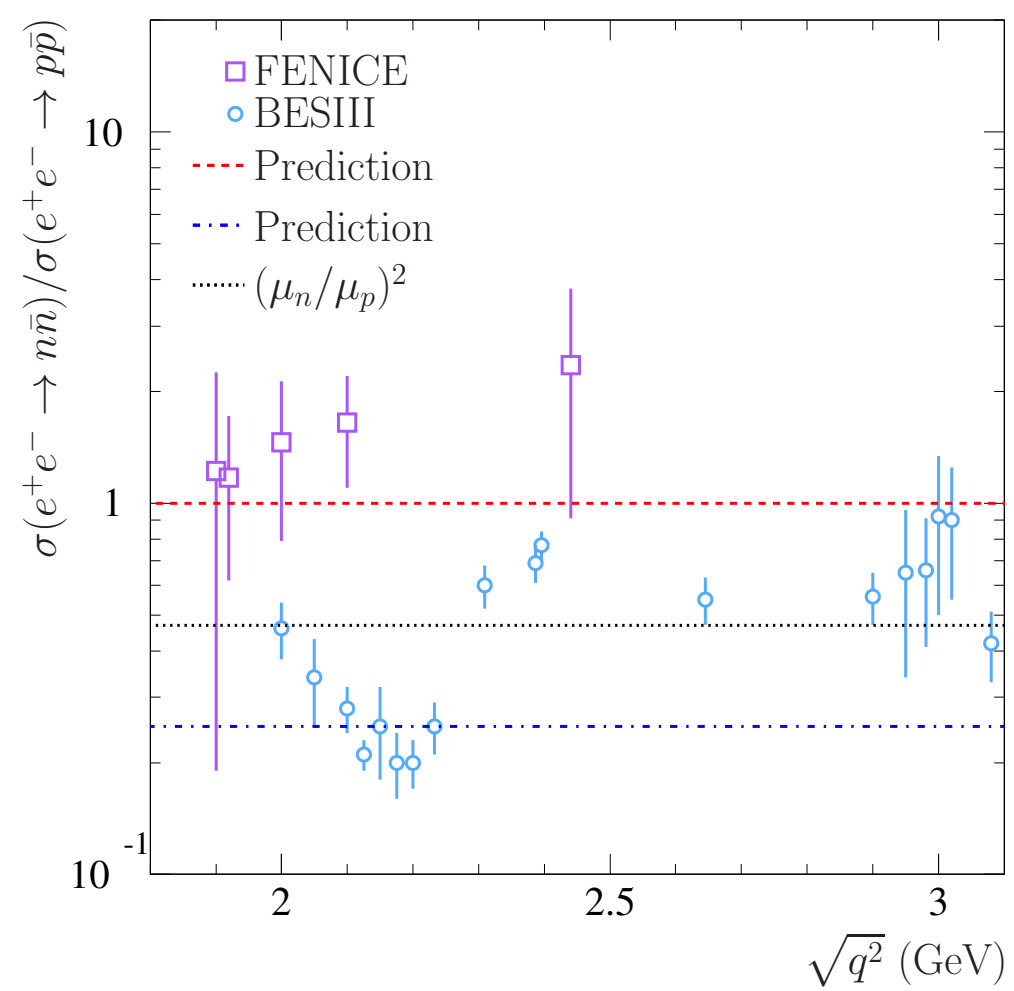

Figure 7. The neutron-to-proton cross-section ratio [15] measured by BESIII and FENICE, together with two theoretical predictions $[1,31]$ and, with the black dotted horizontal line, the squared ratio between the neutron and proton magnetic momenta.

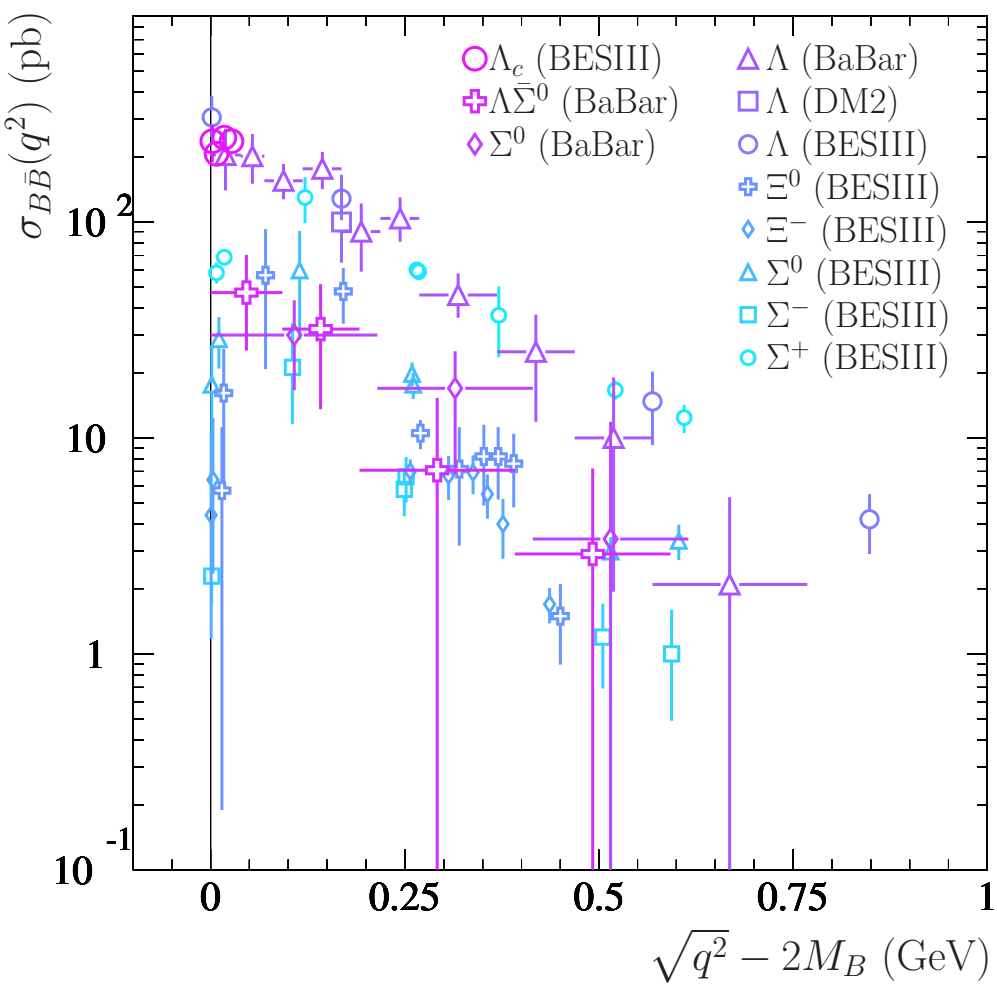

Figure 8. Data on the $e^{+} e^{-} \rightarrow B \bar{B}$ cross-section measured by the experiments: BESIII [32-37], BABAR [38], and DM2 [26], where $B \bar{B}=\Lambda_{c} \bar{\Lambda}_{c}, \Lambda \bar{\Sigma}^{0}+$ c.c., $\Sigma^{0} \bar{\Sigma}^{0}, \Lambda \bar{\Lambda}, \Xi^{0} \bar{\Xi}^{0}, \Xi^{-} \bar{\Xi}^{+}, \Sigma^{-} \bar{\Sigma}^{+}, \Sigma^{+} \bar{\Sigma}^{-}$. 


\title{
6. Conclusions
}

In this review, we discussed the theoretical properties of baryons' form factors, by placing a particular emphasis on the connection between time-like and space-like regions, and hence on the possibilities of relating FFs values extracted from cross-section data on annihilation and scattering reactions, respectively.

In this respect, we briefly introduced dispersion relations, being one of the most powerful mathematical tools to perform the analytic continuation from the time-like to space-like region.

The time-like-space-like connection, i.e., the possibility of attaining a unique description of FFs that is valid at all $q^{2}$, based on data and first-principles features, was also explored by considering other observables such as the baryons' polarization and the root-mean-squared radii.

Finally, we presented all the available data on the most relevant and extensively studies FFs, i.e., those of the nucleons.

\begin{abstract}
Author Contributions: Conceptualization, M.B., A.M. and S.P.; methodology, M.B., A.M. and S.P.; software, M.B., A.M. and S.P.; validation, M.B., A.M. and S.P.; formal analysis, M.B., A.M. and S.P.; investigation, M.B., A.M. and S.P.; resources, M.B., A.M. and S.P.; data curation, M.B., A.M. and S.P.; writing — original draft preparation, M.B., A.M. and S.P.; writing—review and editing, M.B., A.M. and S.P.; visualization, M.B., A.M. and S.P.; supervision, M.B., A.M. and S.P.; project administration, M.B., A.M. and S.P.; funding acquisition, M.B., A.M. and S.P. All authors have read and agreed to the published version of the manuscript.
\end{abstract}

Funding: This work was supported in part by the STRONG-2020 project of the European Union's Horizon 2020 Research and Innovation Programme under Grant Agreement No. 824093.

Institutional Review Board Statement: Not applicable.

Informed Consent Statement: Not applicable.

Conflicts of Interest: The authors declare no conflict of interest.

\section{References}

1. Pacetti, S.; Ferroli, R.B.; Tomasi-Gustafsson, E. Proton electromagnetic form factors: Basic notions, present achievements and future perspectives. Phys. Rept. 2015, 550-551, 1-103. [CrossRef]

2. Hofstadter, R.; McAllister, R.W. Electron Scattering from the Proton. Phys. Rev. 1955, 98, 217-218. [CrossRef]

3. Yearian, M.R.; Hofstadter, R. Magnetic Form Factor of the Neutron. Phys. Rev. 1958, 110, 552. [CrossRef]

4. Alberto, P.; Drago, A.; Mangoni, A.; Moretti, S.; Pacetti, S. Analytic continuation of nucleon electromagnetic form factors in the time-like region. J. Phys. G 2021, 48, 085007. [CrossRef]

5. Titchmarsh, E.C. Theory of Functions; Oxford University Press: London, UK, 1939.

6. Pacetti, S.; Tomasi-Gustafsson, E. The origin of the proton radius puzzle. Eur. Phys. J. A 2021, 57, 72. [CrossRef]

7. Gourdin, M. Weak and Electromagnetic Form-Factors of Hadrons. Phys. Rept. 1974, 11, 29. [CrossRef]

8. Mangoni, A.; Pacetti, S.; Tomasi-Gustafsson, E. First exploration of the physical Riemann surfaces of the ratio GE $\Lambda / G M \Lambda$. Phys. Rev. D 2021, 104, 116016. [CrossRef]

9. Fäldt, G.; Kupsc, A. Hadronic structure functions in the $e^{+} e^{-} \rightarrow \bar{\Lambda} \Lambda$ reaction. Phys. Lett. B 2017, 772, 16-20. [CrossRef]

10. Granados, C.; Leupold, S.; Perotti, E. The electromagnetic Sigma-to-Lambda hyperon transition form factors at low energies. Eur. Phys. J. A 2017, 53, 117. [CrossRef]

11. Xia, L.; Rosner, C.; Wang, Y.; Zhou, X.; Maas, F.E.; Ferroli, R.B.; Hu, H.; Huang, G. Proton Electromagnetic Form Factors in the Time-like Region through the Scan Technique. Symmetry 2022, 14, 231. [CrossRef]

12. Lin, D.; Dbeyssi, A.; Maas, F. Time-like Proton Form Factors with Initial State Radiation Technique. Symmetry 2022, $14,91$. [CrossRef]

13. Ablikim, M.; Achasov, M.; Adlarson, P.; Ahmed, S.; Albrecht, M.; Alekseev, M.; Amoroso, A.; An, F.; An, Q.; Anita; et al. Measurement of proton electromagnetic form factors in $e^{+} e^{-} \rightarrow p \bar{p}$ in the energy region 2.00-3.08 GeV. Phys. Rev. Lett. 2020, 124, 042001. [CrossRef] [PubMed]

14. Tomasi-Gustafsson, E.; Bianconi, A.; Pacetti, S. Dynamical Properties of Baryons. Symmetry 2021, 13, 1480. [CrossRef]

15. Ablikim, M.; Achasov, M.N.; Adlarson, P.; Ahmed, S.; Albrecht, M.; Aliberti, R.; Amoroso, A.; An, Q.; Lavania, A.; Bai, X.H.; et al. Oscillating features in the electromagnetic structure of the neutron. Nat. Phys. 2021, 17, 1200-1204. [CrossRef]

16. Armstrong, T.A.; E760 Collaboration. Measurement of the proton electromagnetic form-factors in the timelike region at from 8.9 to $13.0 \mathrm{GeV}^{2}$. Phys. Rev. Lett. 1993, 70, 1212-1215. [CrossRef] 
17. Ambrogiani, M.; Bagnasco, S.; Baldini, W.; Bettoni, D.; Borreani, G.; Buzzo, A.; Calabrese, R.; Cester, R.; Dalpiaz, P.; Fan, X.; et al. Measurements of the magnetic form-factor of the proton in the timelike region at large momentum transfer. Phys. Rev. D 1999, 60, 032002. [CrossRef]

18. Andreotti, M.; Bagnasco, S.; Baldini, W.; Bettoni, D.; Borreani, G.; Buzzo, A.; Calabrese, R.; Cester, R.; Cibinetto, G.; Dalpiaz, P.; et al. Measurements of the magnetic form-factor of the proton for timelike momentum transfers. Phys. Lett. B 2003, 559, 20-25. [CrossRef]

19. Ablikim, M.; Bai, J.Z.; Ban, Y.; Bian, J.G.; Cai, X.; Chen, H.F.; Chen, H.S.; Chen, H.X.; Chen, J.C.; Chen, J.; et al. Measurement of the cross-section for $e^{+} e^{-} \rightarrow p \bar{p}$ at center-of-mass energies from 2.0 to $3.07 \mathrm{GeV}$. Phys. Lett. B 2005, 630, 14-20. [CrossRef]

20. Aubert, B.; Barate, R.; Boutigny, D. A Study of $e^{+} e^{-} \rightarrow p \bar{p}$ using initial state radiation with BABAR. Phys. Rev. D 2006, 73, 012005. [CrossRef]

21. Lees, J.P.; Poireau, V.; Tisserand, V.; Grauges, E.; Palano, A.; Eigen, G.; Stugu, B.; Brown, D.N.; Kerth, L.T.; Kolomensky, Y.G.; et al Study of $e^{+} e^{-} \rightarrow p \bar{p}$ via initial-state radiation at BABAR. Phys. Rev. D 2013, 87, 092005. [CrossRef]

22. Akhmetshin, R.R.; Amirkhanov, A.N.; Anisenkov, A.V.; Aulchenko, V.M.; Banzarov, V.S.; Bashtovoy, N.S.; Berkaev, D.E.; Bondar, A.E.; Bragin, A.V.; Eidelman, S.I.; et al. Study of the process $e^{+} e^{-} \rightarrow p \bar{p}$ in the c.m. energy range from threshold to $2 \mathrm{GeV}$ with the CMD-3 detector. Phys. Lett. B 2016, 759, 634-640. [CrossRef]

23. Antonelli, A.; Baldini, R.; Benasi, P.; Bertani, M.; Biagini, M.E.; Bidoli, V.; Bini, C.; Bressani, T.; Calabrese, R.; Cardarelli, R.; et al The first measurement of the neutron electromagnetic form-factors in the timelike region. Nucl. Phys. B 1998, 517, 3-35. [CrossRef]

24. Bardin, G.; Burgun, G.; Calabrese, R.; Capon, G.; Carlin, R.; Dalpiaz, P.; Dalpiaz, P.F.; Derré, J.; Dosselli, U.; Duclos, J.; et al. Determination of the electric and magnetic form-factors of the proton in the timelike region. Nucl. Phys. B 1994, 411, 3-32. [CrossRef]

25. Bisello, D.; Limentani, S.; Nigro, M.; Pescara, L.; Posocco, M.; Sartori, P.; Augustin, J.E.; Busetto, G.; Cosme, G.; Couchot, F.; et al. A Measurement of $e^{+} e^{-} \rightarrow \bar{p} p$ for $(1975 \leq s \leq 2250) \mathrm{MeV}$. Nucl. Phys. B 1983, 224, 379. [CrossRef]

26. Bisello, D.; Busetto, G.; Castro, A.; Nigro, M.; Pescara, L.; Posocco, M.; Sartori, P.; Stanco, L.; Antonelli, A.; Biagini, M.E.; et al. Baryon pair production in $e^{+} e^{-}$annihilation at $\sqrt{s}=2.4 \mathrm{GeV}$. Z. Phys. C 1990, 48, 23-28. [CrossRef]

27. Antonelli, A.; Baldini, R.; Bertani, M.; Biagini, M.E.; Bidoli, V.; Bini, C.; Bressani, T.; Calabrese, R.; Cardarelli, R.; Carlin, R.; et al. Measurement of the electromagnetic form-factor of the proton in the timelike region. Phys. Lett. B 1994, 334, 431-434. [CrossRef]

28. Biagini, M.E.; Pasqualucci, E.; Rotondo, A. U spin considerations to guess the unknown timelike neutron form-factors. Z. Phys. C 1991, 52, 631-634. [CrossRef]

29. Achasov, M.N.; Barnyakov, A.Y.; Beloborodov, K.I.; Berdyugin, A.V.; Berkaev, D.E.; Bogdanchikov, A.G.; Botov, A.A.; Dimova, T.V.; Druzhinin, V.P.; Golubev, V.B.; et al. Study of the process $e^{+} e^{-} \rightarrow n \bar{n}$ at the VEPP-2000 $e^{+} e^{-}$collider with the SND detector. Phys. Rev. D 2014, 90, 112007. [CrossRef]

30. Druzhinin, V.P.; Serednyakov, S.I. Measurement of the $e^{+} e^{-} \rightarrow n \bar{n}$ cross-section with the SND detector at the VEPP-2000 collider. EPJ Web Conf. 2019, 212, 07007. [CrossRef]

31. Chernyak, V.L.; Zhitnitsky, A.R. Asymptotic Behavior of Exclusive Processes in QCD. Phys. Rept. 1984, 112, 173. [CrossRef]

32. Ablikim M.; Achasov M.N.; Adlarson P.; Ahmed S.; Albrecht M.; Aliberti R.; Amoroso A.; An Q.; Bai X.H.; Bai Y.; et al Measurement of cross-section for $e^{+} e^{-} \rightarrow \Xi^{-} \bar{\Xi}^{+}$near threshold at BESIII. Phys. Rev. D 2021, 103, 012005. [CrossRef]

33. Ablikim, M.; Achasov, M.N.; Adlarson, P.; Ahmed, S.; Albrecht, M.; Aliberti, R.; Amoroso, A.; An, M.R.; An, Q.; Ba, X.H.; et al. Measurement of cross-section for $e^{+} e^{-} \rightarrow \Xi^{0} \bar{\Xi}^{0}$ near threshold. Phys. Lett. B 2021, 820, 136557. [CrossRef]

34. Ablikim, M.; Achasov, M.; Ahmed, S.; Albrecht, M.; Alekseev, M.; Amoroso, A.; An, F.; An, Q.; Bai, J.; Bai, Y.; et al. Precision measurement of the $e^{+} e^{-} \rightarrow \Lambda_{c}^{+} \bar{\Lambda}_{c}^{-}$cross-section near threshold. Phys. Rev. Lett. 2018, 120, 132001. [CrossRef] [PubMed]

35. Ablikim, M.; Achasov, M.; Ahmed, S.; Ai, X.; Albayrak, O.; Albrecht, M.; Ambrose, D.; Amoroso, A.; An, F.; An, Q.; et al. Observation of a cross-section enhancement near mass threshold in $e^{+} e^{-} \rightarrow \Lambda \bar{\Lambda}$. Phys. Rev. D 2018, 97, 032013. [CrossRef]

36. Ablikim, M.; Achasov, M.N.; Adlarson, P.; Ahmed, S.; Albrecht, M.; Amoroso, A.; An, Q.; Anita; Bai, Y.; Bakina, O.; et al. Measurements of $\Sigma^{+}$and $\Sigma^{-}$time-like electromagnetic form factors for center-of-mass energies from 2.3864 to $3.0200 \mathrm{GeV}$. Phys. Lett. B 2021, 814, 136110. [CrossRef]

37. Ablikim, M.; Achasov, M.N.; Adlarson, P.; Ahmed, S.; Albrecht, M.; Amoroso, A.; An, Q.; Anita; Bai, Y.; Bakina, O.; et al. Measurement of the $e^{+} e^{-} \rightarrow \Sigma^{0} \bar{\Sigma}^{0}$ cross-sections at center-of-mass energies from 2.3864 to $3.0200 \mathrm{GeV}$. arXiv 2021, arXiv:2110.04510.

38. Ablikim, M.; Achasov, M.N.; Adlarson, P.; Ahmed, S.; Albrecht, M.; Amoroso, A.; An, Q.; Anita; Bai, Y.; Bakina, O.; et al. Study of $e^{+} e^{-} \rightarrow \Lambda \bar{\Lambda}, \Lambda \bar{\Sigma}^{0}, \Sigma^{0} \bar{\Sigma}^{0}$ using initial state radiation with BABAR. Phys. Rev. D 2007, 76, 092006. [CrossRef] 